\title{
Pour une meilleure connaissance des techniques agricoles anciennes de Bretagne. La lexicographie au service de la technologie
}

Towards a better knowledge of Brittany's ancient agricultural techniques:

lexicography shedding light on technology

Jean-François Simon

\section{(2) OpenEdition \\ Journals}

Édition électronique

URL : https://journals.openedition.org//bl/1583

DOl : 10.4000/lbl.1583

ISSN : 2727-9383

\section{Éditeur}

Université de Bretagne Occidentale - UBO

\section{Édition imprimée}

Date de publication : 1 février 2013

Pagination : 65-79

ISBN : 979-10-92331-00-4

ISSN : $1270-2412$

\section{Référence électronique}

Jean-François Simon, «Pour une meilleure connaissance des techniques agricoles anciennes de Bretagne. La lexicographie au service de la technologie », La Bretagne Linguistique [En ligne], 17 | 2013, mis en ligne le 01 mai 2021, consulté le 22 mai 2021. URL : http://journals.openedition.org//bl/1583 DOI : https://doi.org/10.4000/lbl.1583

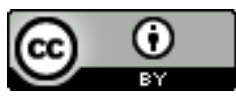

La Bretagne Linguistique est mise à disposition selon les termes de la Licence Creative Commons Attribution 4.0 International. 
Jean-François SIMON*

\section{Pour une meilleure connaissance des techniques agricoles anciennes de Bretagne. La lexicographie au service de la technologie}

\section{L}

es spécialistes de l'histoire ${ }^{1}$ des techniques agricoles s'entendent pour dire que la littérature française (et donc probablement bretonne) contemporaine fait un usage souvent incorrect des termes techniques relatifs aux pratiques agricoles : une illustration peut en être donnée à propos du sillon, ero en breton. Ce mot est aujourd'hui utilisé pour signifier la trace en creux laissée par la charrue. Or cette trace est en fait la raie, ant en breton, tandis que le sillon doit être compris comme étant la terre retournée par plusieurs passages successifs de l'instrument aratoire. À titre d'exemple, à Plouzané, on dit :

Un ero a ra c'hwec'h bomm

Un sillon fait six passages [de charrue]

* Professeur d'ethnologie, CRBC (EA 4451), UBO/ueb

1. Un terme qui, à l'instar du positionnement épistémologique de François Sigaut, inclut les autres sciences humaines, telles que la géographie, l'ethnologie, la linguistique etc. (cf. François Sigaut, «Le labour, qu'est-ce que c'est ?», dans René BourRIGAUd et François Sigaut (dir.), Nous labourons, Nantes, Éditions du Centre d'histoire du travail, 2007, p. 21-27, p. 21. 
Sommairement, on peut dire que le sillon ainsi compris constitue la partie bombée créée lors du labour, qui justifie qu'il puisse y avoir par exemple, sur le littoral des Côtes d'Armor un sillon de Talbert, de galets celui-ci, qui soit toujours émergé.

Inutile de s'appesantir sur les possibles erreurs d'interprétation qui peuvent en résulter, d'autant que le mot sillon peut en outre désigner à la fois le résultat d'une action de labourage comme on vient de le voir, et une unité de surface, l'un et l'autre exprimés en valeurs qui ne sont pas exactement confondues.

De ces approximations, il découle que pour parvenir à une juste connaissance de tels termes techniques et à leur emploi approprié, les spécialistes se tournent d'abord vers les «grands ancêtres ${ }^{2}{ }^{2}$ » de l'agronomie européenne, leur reconnaissant «un esprit d'observation et un jugement sûrs ${ }^{3}$ » qui en font des témoins crédibles. Ce sont aussi ces observateurs précis qui sont dans le même temps responsables du passage de certains termes techniques vernaculaires dans le langage français, d'abord savant puis courant.

Mais, ce faisant, ou les dits agronomes ne rapportent que des termes en usage dans les langues romanes, ou ils interprètent des termes bretons qu'ils ont pu entendre, avec la part d'imprécisions que ceci comporte. De leur côté, les technologues que ces questions intéressent, ne se sont pas particulièrement souciés, à ma connaissance, d'étudier les mots bretons exprimant de telles réalités techniques. C'est à ce titre que les lexicographes peuvent être convoqués : ils ont en effet établi des dictionnaires breton-français ou français-breton dont la consultation permet de pallier les carences éventuelles des "grands ancêtres » et d'apporter à l'occasion des éléments nouveaux d'appréciation pouvant éclairer le sujet. Les quelques lexicographes que j'ai consultés sont Grégoire de Rostrenen ${ }^{4}$, Louis Le Pelletier

2. François Sigaut, L'agriculture et le feu. Rôle et place du feu dans les techniques de préparation du champ de l'ancienne agriculture européenne, Paris, EHESS La Haye - Mouton et $\mathrm{C}^{\circ}, 1975$, p. 5.

3. ID., p. 6.

4. Dictionnaire français-celtique ou français-breton, Guingamp, Benjamin Jollivet, 1834 [1732]. 
(versions manuscrite ${ }^{5}$ et imprimée ${ }^{6}$ ), Le Gonidec ${ }^{7}$ dont les informations ont été complétées par des notes extraites du Dictionnaire manuscrit en huit volumes de de Coëtanlem ${ }^{8}$.

Pour aller plus avant dans cette direction, j'ai pris le parti d'examiner le cas de la houe en Bretagne. François Sigaut, directeur d'études à l'École des hautes études en sciences sociales (EHESS), spécialiste reconnu de ces questions, dit en effet que «les houes de Bretagne posent bien des problèmes... ${ }^{9} \gg$. Paradoxalement d'ailleurs, ces problèmes surgissent à la lecture des descriptions données par les agronomes, qu'il s'agisse de l'outil en question ou de l'utilisation qui en est faite. Je me suis donc demandé si les lexicographes bretons ne pouvaient pas venir au secours du technologue pour tenter d'y voir plus clair.

\section{Selon André Leroi-Gourhan,}

La houe est certainement le plus important des instruments aratoires, le seul qui manifeste une haute densité dans toutes les parties du monde, depuis les débuts de l'agriculture et aussi bien chez les peuples rustiques que chez nous c'est en effet l'objet le plus propre à travailler la terre à la main ${ }^{10}$.

La houe peut avoir des formes et des tailles variées ; André Leroi-Gourhan la classe dans les outils à percussion lancée. Dans la littérature française actuelle, y compris savante, elle est présentée comme étant, soit une écobue soit une étrèpe.

5. Dictionnaire de la langue bretonne suivi de textes littéraires et de quelques études, Rennes, Bibliothèque municipale, 1975 [manuscrit de 1716].

6. Dictionnaire de la langue bretonne, Paris, Delaguette, 1752.

7. Dictionnaire breton-français, Saint-Brieuc, Prud'homme, 1850.

8. Dictionnaire [...], par Dom Louis Le Pelletier [...] ; et c'est d'après cette édition que le présent manuscrit a été dressé avec des additions et remarques par Monsieur de Coëtanlem, en son château de Trogriffon, près Morlaix. Pierre Joseph Jean chevalier de Coëtanlem de Rostiviec est né à Saint-Martin-desChamps en 1749 et décédé à Henvic en 1827.

9. François Sigaut, L'agriculture et le feu..., op. cit., p. 61.

10. André Leroi-Gourhan, Milieu et techniques, Paris, Albin Michel, 1973 [1945], p. 120 . 


\section{Une écobue?}

Selon les agronomes du XVIII siècle, "égobuer les terres ${ }^{11} »$, c'est, dans la France de l'Ouest et du Centre, procéder au dégazonnement d'une parcelle pour la remettre en culture. Cette action produit des mottes qui sont alors mises à sécher pour être ensuite brûlées sur place ; les cendres qui en résultent sont utilisées comme fertilisant : cette opération est connue sous le nom d'écobuage, depuis l'introduction du mot dans le français littéraire, à une date qui est estimée se situer vers $1760^{12}$.

À la même époque, en Bretagne, l'opération est connue sous le nom d'écobue, comme en attestent Grégoire de Rostrenen et Louis Le Pelletier lequel explique en outre, dans une phrase qui me paraît quelque peu énigmatique à moins qu'elle ne soit mal tournée, que «ceux [des Armoricains] qui parlent françois, \& ont conservé plusieurs mots Bretons, disent Ecaubuë au sens de Marradec ; \& Escaubuer pour Marra, travailler de la marre.» (p. 582).

D'après François Sigaut, le mot «écobue», pensé comme désignant l'outil servant à exécuter l'opération du même nom aujourd'hui désignée par écobuage, est un mot qui a été introduit dans le français littéraire par Louis François Henri de Menon, marquis de Turbilly, qui l'aurait entendu dire en Anjou ${ }^{13}$. François Sigaut doute cependant de l'appartenance du mot écobue «au langage technique populaire véritable de $1^{\prime} A$ njou ${ }^{14} \gg$. Il voit à cela deux raisons :

La première, c'est que dans toute la France, les auteurs donnent en général aux houes à écobuer des noms comme tranches, tranque, treinchée, marre, fessou, foussou, etc., qui ne sont autres

11. Henri Louis Duhamel du Monceau, Éléments d'agriculture, Paris, 1762 ; cité par François Sigaut, L'agriculture et le feu..., op. cit., p. 11.

12. Dans la logique de ce qui a été annoncé plus haut, cette opération agricole est aujourd'hui mal comprise et renvoie à un brûlage à feu courant, tel qu'il est pratiqué en Corse et dans le sud de la France et qui vaut à ceux qui le pratiquent d'être à l'occasion conduits devant les tribunaux.

13..Mémoire sur les défrichemens, Paris, 1761.

14. François Sigaut, L'agriculture et le feu..., op. cit., p. 56. 
que des noms de houes ordinaires ${ }^{15}:$ le nom très spécialisé de l'écobue d'Anjou serait ainsi une exception. La seconde raison, c'est qu'écobue a aussi le sens de terre écobuée (quoiqu'au pluriel seulement : écobues), qui ferait double emploi avec le premier. Des précisions seraient nécessaires sur ce point ${ }^{16}$.

Quelques "grands ancêtres» ont fourni des descriptions et même donné des illustrations de l'outil servant à "égobuer» : ainsi le marquis de Turbilly lui-même et Duhamel de Monceau ${ }^{17}$. Dans son ouvrage consacré à L'agriculture et le feu dans l'ancienne agri-
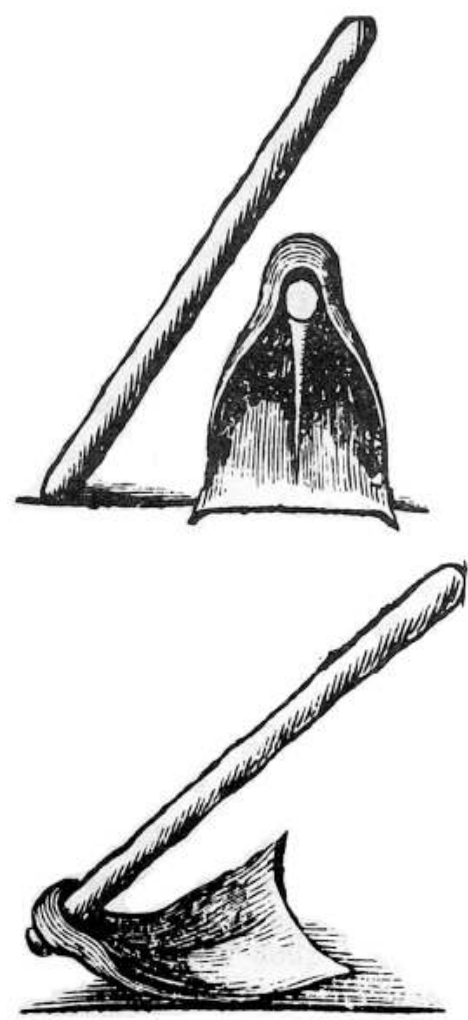
culture européenne, François Sigaut a reproduit ces dessins avec les commentaires qui les accompagnaient: le marquis de Turbilly le décrit comme «une espèce de tranche recourbée, de seize pouces de long et de huit pouces et demi de large par en bas, d'où la largeur va toujours en diminuant jusqu'auprès du manche, où elle se trouve réduite à trois pouces » (fig. 1), Duhamel de Monceau en parle comme d' «une pioche courbe dont le fer est large et mince» (fig. 2).

Fig. 1 : «Une espèce de tranche recourbée, de seize pouces de long et de huit pouces et demi de large par en bas, d'où la largeur va toujours en diminuant jusqu'auprès du manche, où elle se trouve réduite à trois pouces", Louis $\mathrm{F}$. $\mathrm{H}$. de MENON, marquis de TURBILLY, Mémoire sur les défrichemens, Paris, 1761 (cf. François SIGAUT, L'agriculture et le feu. op. cit., p. 55 pour la citation, p. 58 pour le croquis).

15. À condition toutefois de retenir que la «marre» puisse être considérée comme une houe ordinaire, ce qui n'est probablement pas le cas, si l'on se réfère justement aux explications données par les lexicographes bretons.

16. François Sigaut, L'agriculture et le feu ..., op. cit., p. 56.

17. Cf. François Sigaut, L'agriculture et le feu..., op. cit., p. 13 et 58. 


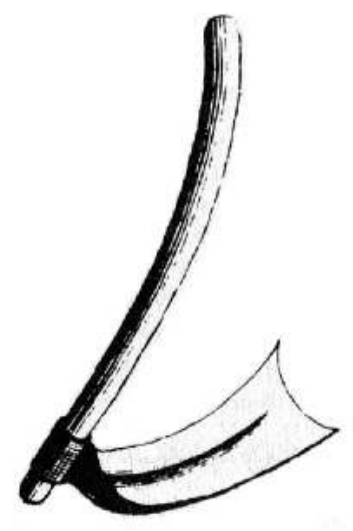

Fig. 2 : «Pioche courbe dont le fer est mince et large», H.L. DuHAmel du MONCEAU, Élémens d'agriculture, Paris, 1762 (cf. François SIGAUT, L'agriculture et le feu, op. cit., p. 11 pour la citation, p. 13 pour l'illustration).

En Bretagne bretonnante, le nom donné à l'outil qui sert à écobuer est marr, en français marre : c'est une «grande houe» précise Grégoire de Rostrenen, dont on se sert pour «marrer, écobuer, peler la terre, marra, marrat» (II, p. 123) et cela lors d'une «marrerie», entendue comme étant une «action de marrer, [un] jour de réjouissance après la besogne, marradeg, pl. marradegou» (id.); de son côté, Louis Le Pelletier explique que la marre est un «outil qui tranche et enlève la croûte de la terre ${ }^{18} »$; de Coëtanlem pour sa part est plus précis, qui en donne quelques caractéristiques techniques :

L'instrument que nous appelons Marr est une pièce de fer forgé, large, plate, tranchante et d'une forme arrondie, terminée par un anneau très fort avec lequel elle forme un angle, au moyen de quoi elle se trouve recourbée sous le long manche introduit dans cet anneau. En travaillant avec cet instrument on tire à soi les mottes qu'on enlève de la superficie de la terre. (Volume V, p. 185.)

Enfin, selon Le Gonidec, la marre est une «espèce de grande houe servant à écroûter la terre pour en brûler les mottes» (p. 434), signifiant donc par cette dernière précision, qu'il s'agit de l'outil de l'écobuage. La description donnée par de Coëtanlem, d'une houe ronde, est notamment confirmée par un dessin d'Olivier Perrin, datable de la même époque (fig. 3).

18. Dans l'article «Marrbikell» du manuscrit (p. 914). 


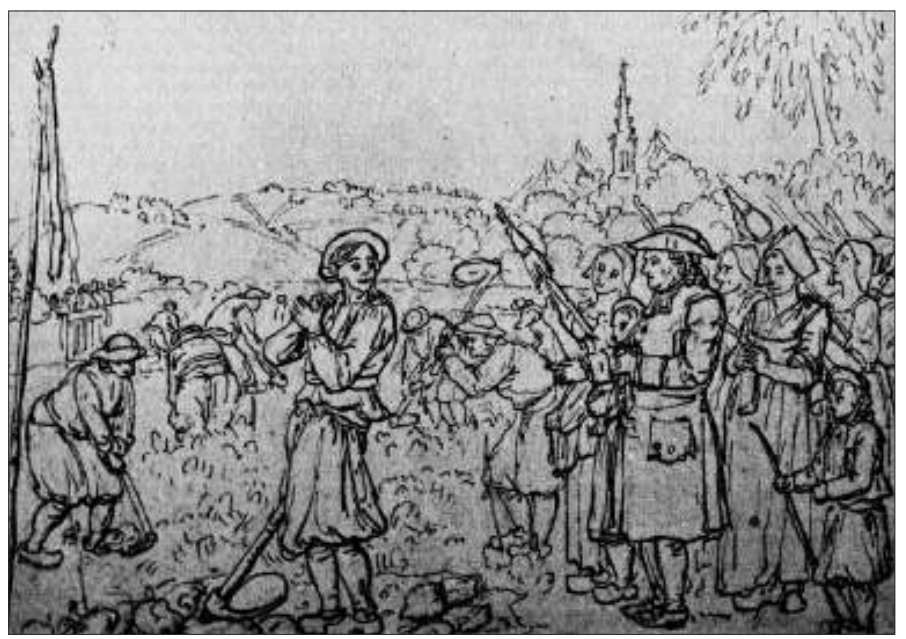

Fig. 3 : Breiz-Izel ou vie des Bretons de l'Armorique, texte d'Alexandre Bouët, dessins d'Olivier Perrin, présentés et commentés par Mr le Médecin Général Ch. Laurent, Mayenne, Joseph Floch - Quimper, Société archéologique du Finistère, 1977, p. 135.

Ainsi, si l'on se réfère aux lexicographes breton, la marre est bien la houe qui sert à l'écobuage. De Coëtanlem donne par ailleurs quelques indications sur l'opération qui justifie son utilisation :

Dans ce païs c'est la première opération que l'on fait pour disposer la terre où l'on doit ensemencer du seigle, de la lande, $\&$. Ce genre de travail, qu'on appelle Marrerez est très dur, et le partage des hommes les plus robustes ; aussi le PG [père Grégoire (de Rostrenen)] n'a pas donné de féminin à Marrer, Marreur. Ce féminin serait régulièrement Marreres, pl. Marreresed, mais il n'est guère en usage, parce que les femmes ne se mêlent pas de l'ouvrage [...] quoiqu'elles travaillent souvent avec la houe ou de vieilles marres usagées, pour rompre les mottes et rendre la terre plus meuble mais lorsqu'on se propose de marrer une pièce de terre d'une certaine étendue, on réunit autant qu'on peut un grand nombre d'hommes et de jeunes gens vigoureux qui travaillent à l'envi. C'est cette réunion, ce rassemblement, l'opération qui en est le résultat et la fête ou réjouissance qui termine la besogne, comme l'observe le PG qu'on appelle Marradeg. (Vol. V, page 186.) 


\section{Une étrèpe ?}

La question de l'étrèpe est plus complexe. Sa description et son usage ont donné lieu à nombre d'approximations qui troublent l'approche que l'on peut en avoir aujourd'hui. Là encore, les lexicographes bretons peuvent-ils aider à y voir plus clair?

Selon François Sigaut, «en français courant, étrèpage vient indubitablement de l'expression bretonne, rapportée pour la première fois en 1807 par de Candolle ${ }^{19} \gg$ alors que ce dernier effectuait une mission agro-botanique dans l'Ouest de la France. De Candolle écrit en effet : «Les instruments réellement propres à la Bretagne sont les étrèpes ou streps, parce que l'opération qui les a rendu nécessaires est heureusement bornée à ce pays ${ }^{20} \gg$. Il existe bien une entrée «Etrape» dans le dictionnaire français-breton de Grégoire de Rostrenen.

Les descriptions qu'en donnent les agronomes manquent singulièrement de précisions. Ainsi de Candolle :

L'étrèpe est une tranche large et courte, dont le bord est fort coupant et dont le manche est disposé de manière à faire avec la lame un angle d'environ 35 degrés. L'étrèpe de Vannes a les deux côtés arrondis et égaux ; celle de Quimper, qui porte le nom de mare [sic], est proportionnellement plus étroite ; celle de Muzillac est très singulière, en ce que l'un des côtés se prolonge en une oreillette aiguë ${ }^{21}$.

D’emblée, on notera que, dans la définition qui se veut générale, l'étrèpe est décrite comme ayant une lame «large et courte», différente par conséquent de celle de l'écobue décrite par les agronomes évoqués plus haut, qui est plutôt longue et étroite, en tout cas plus longue que large ; on notera aussi que dans la région de Quimper, la marre ne serait qu'une expression localisée de l'étrèpe.

19. François Sigaut, L'agriculture et le feu..., op. cit., p. 180.

20. De CANDOlle, «Rapport sur un voyage botanique et agronomique dans les départements de l'Ouest», Mémoires d'agriculture, d'économie rurale et domestique publiés par la Société d'agriculture du département de la Seine, Paris, X, cité par François Sigaut, L'agriculture et le feu ..., op. cit., p. 57.

21. Cité par François Sigaut, L'agriculture et le feu..., op. cit., p. 58-59. 
Une autre confusion apparaît dans l'usage qui est fait de cet outil : «Ces étrèpes, poursuit de Candolle, servent à enlever la superficie de la terre végétale qui se forme sur les landes, et à couper le pied des ajoncs et autres arbustes qui s'y sont développés... ${ }^{22} »$. L'objectif d'une telle opération est de produire du fumier : les végétaux ainsi recueillis servent de litière dans les étables ou sont étalés dans les cours de fermes pour être piétinés et recevoir les déjections animales. En d'autres termes, l'action de travailler avec l'étrèpe, du coup désignée par étrépage, tiendrait à la fois d'un écobuage mais sans qu'il y ait brûlage et d'un soutrage, du nom donné à l'opération qui consiste à recueillir des végétaux pour en faire du fumier, dans les conditions qui ont été évoquées plus haut.

À cet égard, une question se pose cependant. Peut-on véritablement réaliser ces deux actions avec un même outil, deux actions qui ne paraissent pas avoir le même enjeu : d'une part, enlever une couche de terre végétale avec les espèces qui y poussent et d'autre part, simplement couper les végétaux à la surface du sol ? Certes, les écobues peuvent trancher les racines, mais sont-elles pour autant propres à couper les tiges aériennes des végétaux ? Inversement, un outil dont le fil de la lame serait assez fin pour sectionner des branches, resterait-il longtemps affûté, s'il devait être mis au contact de la terre et des cailloux qu'elle contient?

Or, il semblerait que l'étrèpe soit à ranger du côté des outils au tranchant acéré : Grégoire de Rostrenen donne en effet au mot «Etrape» la définition d'un «instrument pour couper le chaume, etc.», et le traduit par «strep, pl. ou [c'est le mot rapporté par de Candolle mais dans une forme erronée du pluriel] ; inglod, pl. ou, inglojou ; beon, pl. ou ; fals strop» (I, p. 367). Louis Le Pelletier pour sa part ne mentionne pas les mots inglod ni beon mais définit la fals-strop comme étant «un instrument emmanché dans un long bâton, lequel sert à tailler les hayes \& quelques arbres» (p. 296), précisant à l'article «Strop», pour qualifier la «fals strop» que «c'est une espéce de faucille sans dents avec laquelle on coupe à

22. De CANDolle, «Rapport sur un voyage...», cité par François Sigaut, L'agriculture et le feu..., op. cit., p. 59. 
tour de bras» (p. 846), précisant encore dans la version manuscrite de son dictionnaire, que cette espèce de faucille est «emmanchée au bout d'un gros bâton» (p. 1237), «un long bâton» précise pour sa part de Coëtanlem (volume III, p. 341).

Ce sont là des descriptions d'où il ressort que, paradoxalement, l'étrépage décrit aujourd'hui comme étant l'action qui consiste à enlever les végétaux et leur motte pour en faire du fumier, n'est guère techniquement envisageable avec la mise en œuvre de l'outil appelé étrèpe ou «étrape».

La confusion pourrait rétrospectivement s'expliquer par la ressemblance trompeuse existant entre les deux outils : par exemple, l'angle aigu que forment dans les deux cas la partie travaillante et le manche ; une possible erreur d'interprétation pourrait aussi venir du fait que la gestuelle que nécessite la manipulation des deux outils, la marre (ou écobue) et l'étrèpe, est assez comparable : saisi à deux mains, ils permettent d'opérer une percussion lancée, oblique, linéaire et transversale, mais la matière sur laquelle il y a intervention n'est pas la même : terre et racines dans le cas de la marre (ou de l'écobue), tiges végétales dans le cas de l'étrèpe.

Une telle confusion est apparue très tôt et elle s'est maintenue jusqu'à nos jours...

Les descriptions d'étrèpes sont nombreuses dans la littérature agronomique du $19^{\mathrm{e}}$ siècle. Mais malheureusement, elles semblent avoir toutes été recopiées les unes sur les autres. Leur source unique est probablement le Cours de culture [de Thouïn] ${ }^{23}$, qui ne fut publié qu'en 1827, après sa mort. On y trouve deux dessins d'étrèpe («houe étrapa») [...]. Il semble que la première [fig. 4] soit la «bonne» (c'est-à-dire celle de de Candolle). La seconde [fig. 5] ressemble davantage à une houe ordinaire, voire une écobue : est-ce là l'origine des nombreuses confusions entre les deux outils ? ${ }^{24}$

23. A. ThoüIn, Cours de culture et de naturalisation des végétaux, Paris, 1827.

24. François Sigaut, L'agriculture et le feu..., op. cit., p. 59. 
Fig. 4 : "Houe étrapa de Bretagne», [sert] pour couper entre deux terres les bruyères, les genets et les ajoncs...», d'après A. Thouïn, Cours de culture et de naturalisation des végétaux, Paris, 1827 (cf. François SIGAUT, L'agriculture et le feu. op. cit., p. 58).
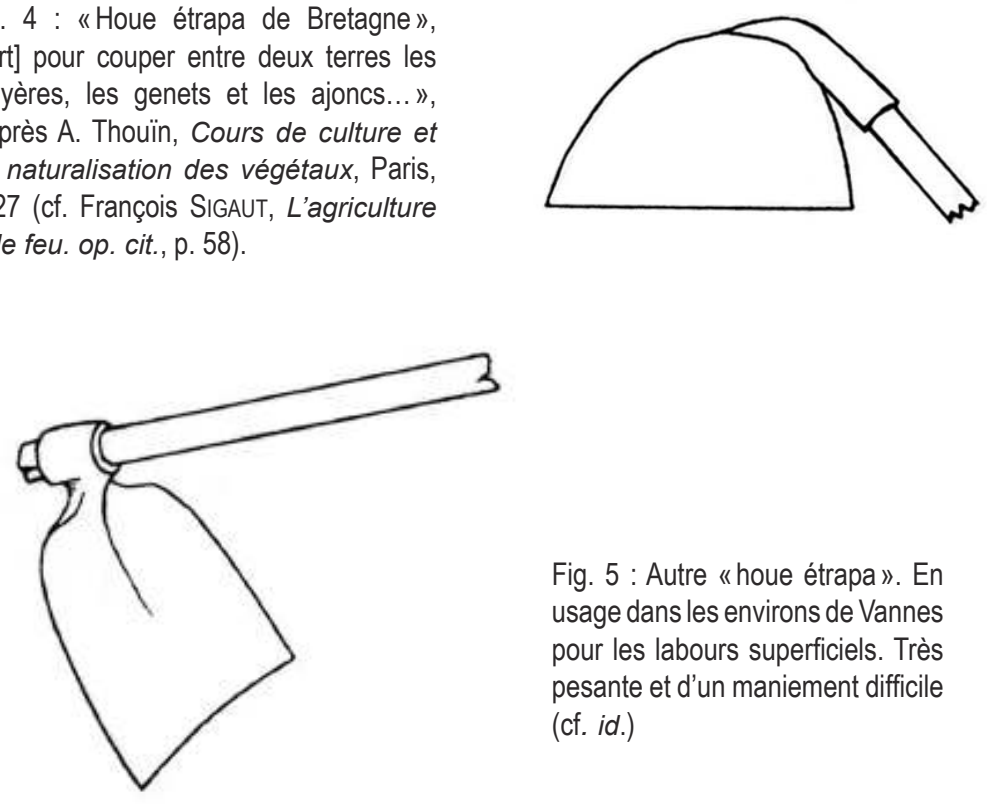

Fig. 5 : Autre «houe étrapa». En usage dans les environs de Vannes pour les labours superficiels. Très pesante et d'un maniement difficile (cf. id.)

Qu'il y ait eu confusion, François Sigaut en convient lui-même après avoir examiné deux outils conservés au Musée national des techniques, outils dont il fournit par ailleurs une illustration (fig. 6) ; il reconnaît volontiers que $« \mathrm{~J}$. Rieffef ${ }^{25}$ avait donc bien raison, lorsqu'il affirmait que l'écobue et l'étrèpe étaient deux outils différents, non substituables l'un à l'autre. ${ }^{26}$ L'examen des renseignements fournis par les lexicographes bretons permet de confirmer ce point de vue. Ce sont autant d'éléments convergents qui permettent finalement d'affirmer que l'étrèpe n'est donc pas une houe, encore moins une marre. Par ailleurs, il ne paraît pas justifié de faire une distinction entre étrépage et soutrage, sauf à faire valoir une spécificité régionale, bretonne en l'occurrence, non pas basée sur une technique opératoire qui serait différente, mais sur une éventuelle originalité de l'outil utilisé, c'est-à-dire l'étrèpe qui serait, sinon inexistant du moins plus rare ailleurs.

25. J. RiEFFEL, «Pratique des défrichements des landes», Agriculture dans l'Ouest de la France, I, 1840.

26. François Sigaut, L'agriculture et le feu..., op. cit., p. 273. 


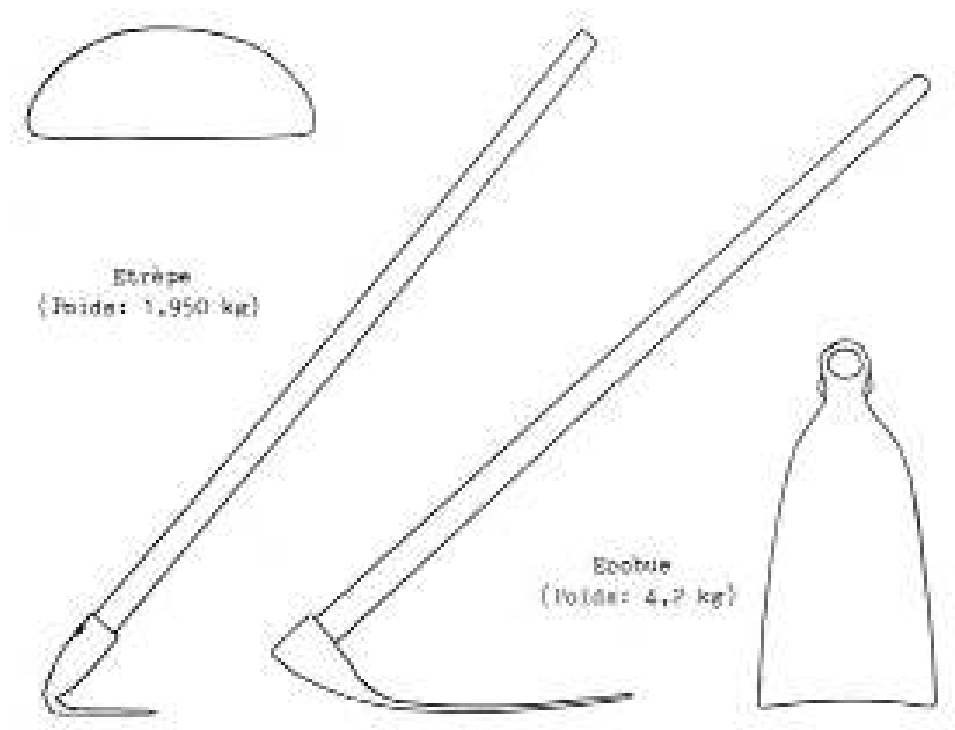

Fig. 6 : Étrèpe et écobue du Musée national des techniques : «On a représenté les outils de profil, les lames vues par leur face inférieure» (cf. François SIGAUT, L'agriculture et le feu. op. cit., p. 275).

Cependant, comme rien n'est jamais simple, à leur tour les lexicographes bretons brouillent les pistes. Ainsi, Grégoire de Rostrenen donne également au mot «Étrape» figurant comme entrée dans son dictionnaire, la traduction bretonne «marbiguell». Or, Louis Le Pelletier fournit à ce mot «Marbikell», la définition suivante :

Autre instrument de laboureur. C'est une sorte de hoiau, fait à peu près comme une marre, dont on se sert pour travailler au guéret [...] La différence qui est entre ce composé [marr et pighell ou pikell] et le simple Marr, est que celui-ci signifie un outil qui tranche et enlève la croûte de la terre, et l'autre marque un instrument qui entre dans la terre comme un pic [\& la tourne (est-il précisé dans la version imprimée du dictionnaire, p. 582)]. Aussi ce nom est composé de Marr \& de Pighel, pioche. (p. 914).

Un même outil qui aurait deux fonctions paraissant incompatibles laisse de Coëtanlem dubitatif :

Le PG au mot Etrape, qu'il définit [comme] un instrument pour couper le chaume, de la bruïere [ce que d'ailleurs G. de 
Rostrenen ne précise pas], \& lui donne en Breton le nom de marbiguell, pl. marbiguellou. (volume V, p. 185.)

De son côté, Pierre Trépos dans ses «Enquêtes sur le vocabulaire breton de la ferme ${ }^{27}$ fournit pour «La houe», outre les mots marr et trañch qu'il dit être les noms les plus communs, les mots «streub, Plélauff, strop, Pluvigner, chtrop, Grandchamp, Colpo, chtrep, Baud, Baden, Arz, chtreb, Mûr» qui ne vont pas sans rappeler les «streps» de de Candolle en 1807. En même temps, il dit avoir relevé «marr (ar varr), pl. mirri» à Colpo, comme à Plougoulm, Plougonvelin, etc. (fig. 7).

Alors, à Colpo ? chtrop ou marr?

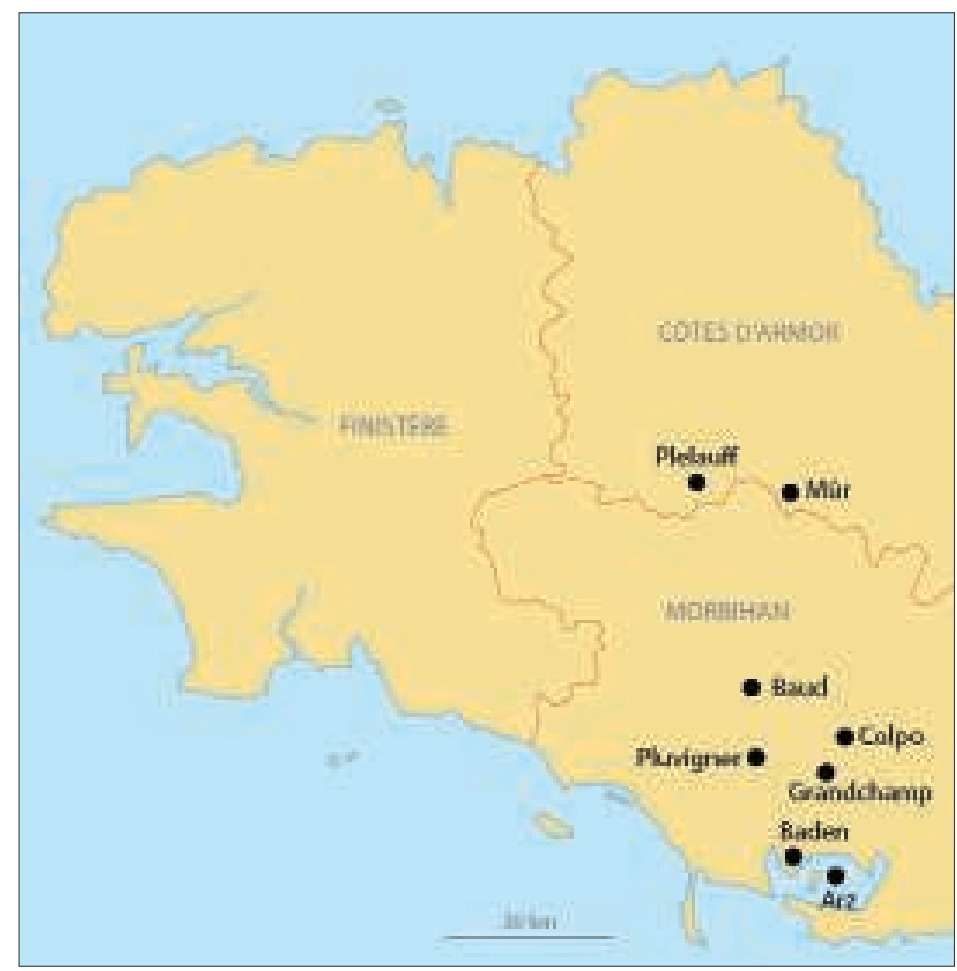

Fig. 7 : Selon Pierre Trépos : localités où la houe se dit streub, strop, chtrop, chtrep, chtreb (carte Gilles Couix). 
On le constate, les houes de Bretagne continuent à poser problème : elles mériteraient en fait une enquête plus poussée. À cet égard, les résultats d'une recherche ponctuelle menée par Claire Dagorne dans les pays de Vilaine, notamment à Bains-sur-Oust et à Béganne, autour de Redon, sont éloquents quant à l'intérêt de mener sur ce sujet une véritable démarche de terrain. Il est ici question de l'étrèpe :

Cet outil se trouve dans toutes les fermes qui ont pratiqué l'élevage $[. .$.$] . Le nom courant est l'étrèpe, qui se prononce$ éteurpe, d'où le verbe éteurper. [...]

C'est un instrument ressemblant à la houe, à la lame recourbée et tranchante, large de plus de 30 centimètres. [...] L'outil était utilisé essentiellement pour éteurper, c'est-à-dire couper la lande qu'on mettait ensuite sous les betes comme litière : les ajoncs au fond [dessous], la bruyère dessus. [...]

Il s'agissait, d'un geste sec vers soi, de couper les végétaux au ras du sol. La bruyère ou les ajoncs bas étaient simplement courbés sous le pied pour dégager la base, puis coupés. [...]

L'éteurpe servait aussi à parer la cour. Pour cela on prenait une éteurpe très usée, ne pouvant guère plus servir à couper la lande. Il s'agissait d'enlever l'herbe et de préparer le sol à l'endroit de la cour de ferme où l'on allait entasser les gerbes de blé avant le battage. Cela se faisait très soigneusement pour obtenir, à petits coups, un sol parfaitement lisse et plat où il serait facile lorsque le blé aurait été battu, de balayer pour recueillir le grain tombé à terre. Le travail pouvait prendre la journée.

Actuellement, pratiquement plus personnes ne se sert de l'éteurpe: on ne va plus couper la lande (la paille a remplacé la lande sous les vaches) et on ne pare plus la cour. Pourtant l'outil est toujours à portée de la main et on le prend parfois pour racler les allées du jardin, afin de les débarrasser des mauvaises herbes.

L'utilisation de l'éteurpe pour couper la lande exigeait un tranchant très effilé et en même temps l'usait beaucoup. Le tranchant était aiguisé régulièrement avant d'aller éteurper, à la ferme, sur la meule : la pierre, la graille (grès). Quand la lame devenait trop courte, on portait l'outil au maréchal à la forge et elle était reforgée en y soudant une lame d'acier trempée, par exemple une vieille lame de ressort.

Avec quelques autres, l'éteurpe était un outil très bien entretenu, bien à la main de son possesseur qui y tenait beaucoup et 
n'aimait guère le prêter. Parfois même, les initiales du propriétaire étaient gravées sur la douille ${ }^{28}$.

Une enquête qui donc laisse entrevoir la manière dont l'étrèpe a pu être considérée comme une houe mais qui montre expressément que son usage n'est pas celui d'un tel outil, sauf à l'avoir détourné de sa destination initiale.

28. Enquête effectuée en 1995 dont les résultats ont été obligeamment communiqués par l'auteure. 